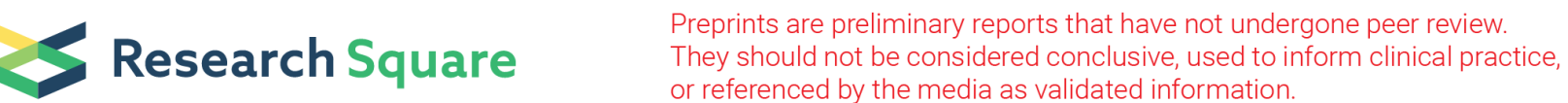

\section{Lymph node yield during surgery after neoadjuvant therapy compared to surgery without neoadjuvant therapy in patients with esophageal, gastric, pancreatic, or rectal carcinoma: systematic review and meta-analysis}

\section{Ulrich Ronellenfitsch}

University Hospital Halle: Universitatsklinikum Halle https://orcid.org/0000-0003-1107-813X

Nika Maximov ( $\nabla$ nika.maximov@student.uni-halle.de )

University Hospital Halle: Universitatsklinikum Halle https://orcid.org/0000-0001-9045-189X

Juliane Friedrichs

University Hospital Halle: Universitatsklinikum Halle

Jörg Kleeff

University Hospital Halle: Universitatsklinikum Halle

\section{Research Article}

Keywords: Lymph node yield, lymph node harvest, neoadjuvant therapy, neoadjuvant chemotherapy, neoadjuvant radiotherapy, surgery, resection, gastrointestinal cancer

Posted Date: April 15th, 2021

DOI: https://doi.org/10.21203/rs.3.rs-421834/v1

License: (c) (1) This work is licensed under a Creative Commons Attribution 4.0 International License. Read Full License 


\section{Abstract}

Background: The lymph node yield is an important surrogate parameter for assessing the oncological radicality of the resection of gastrointestinal carcinomas and a prognostic factor in these diseases. It remains unclear if and to what extent neoadjuvant chemotherapy, radiotherapy or chemoradiotherapy, which have become established treatments for carcinoma of the esophagus, stomach, and rectum and are increasingly used in pancreatic carcinoma, affect the lymph node yield. This systematic review and meta-analysis intents to summarize the available evidence on the topic.

Methods: Studies comparing oncological resection of esophageal, stomach, pancreatic and rectal carcinoma with and without prior neoadjuvant therapy are eligible for inclusion regardless of study design. Publications will be identified with a defined search strategy in the electronic databases PubMed and Cochrane Library. The primary endpoint of the analysis is the number of lymph nodes identified in the resected specimen. Secondary endpoints include number of harvested metastatic lymph nodes, operation time, postoperative complications, pTNM staging, and overall and recurrence-free survival time. Using suitable statistical methods, the endpoints between patients with and without neoadjuvant therapy as well as in defined subgroups (neoadjuvant chemotherapy, neoadjuvant radiotherapy, neoadjuvant chemoradiotherapy, and esophageal, gastric, pancreatic, and rectal cancer) will be compared

Discussion: This systematic review with meta-analysis is conducted with the aim of summarizing the available evidence regarding the oncological surrogate marker lymph node yield in patients with gastrointestinal carcinomas undergoing surgery after neoadjuvant treatment compared to those operated without neoadjuvant therapy.

Systematic review registration: This systematic review has been submitted for registration at PROSPERO, ID: 218459.

\section{Background}

The TNM system for the classification and staging of malignant tumors in its current 8th edition allows for prognostic statements about malignant tumor diseases depending on, among other things, the extent of lymph node involvement (1). The $\mathrm{N}$ category in the TNM classification of gastrointestinal carcinomas is defined by the number of regional lymph nodes with histologically confirmed tumor invasion. Lymphadenectomy, i.e. the systematic resection of the regional lymphatic tissue and lymph nodes, is used for both therapeutic and staging purposes. To allow a valid statement about the number of affected lymph nodes, it is crucial that all regional lymph nodes are removed and identified in the subsequent histopathological examination. Therefore, treatment guidelines often stipulate a minimum number of lymph nodes to be removed and histopathologically analyzed. For example, regarding the surgical treatment of colorectal cancer, the currently valid German S3 guideline specifies that 12 or more lymph nodes be removed and examined (2). Moreover, this practice is supported by the ESMO Clinical Practice 
Guidelines for diagnosis, treatment and follow-up of rectal cancer (3) and the American Society of Colon and Rectal Surgeons Clinical Practice Guidelines for the Treatment of Colon Cancer (4).

However, some studies suggest that neoadjuvant chemotherapy, radiotherapy or chemoradiotherapy, which have become an established treatment for carcinoma of the esophagus, stomach, and rectum, and are increasingly used in resectable pancreatic carcinoma, lower the lymph node yield in the case of colorectal cancer without this having any direct prognostic relevance (5-9). The mechanisms of such lower lymph node yield after neoadjuvant therapy could be based on lymphocyte depletion and radiationinduced fibrosis of the stroma, which lead to a reduction in the size of lymph nodes and thus complicate their surgical and histopathological identification. Moreover, during therapy stromal atrophy and adipocytic replacement occur, which makes lymph node identification more difficult and can thus also contribute to a lower lymph node yield $(10,11)$. This mechanism has been shown especially for radiotherapy and less so for chemotherapy (6). Lastly, although there is no higher level evidence supporting this hypothesis, a more or less aggressive approach and thus a different extent of lymph node dissection employed by the surgeon between patients who underwent neoadjuvant therapy and those who did not may be of importance (12).

Differences in the lymph node yield possibly lead to understaging with regard to lymph node $(\mathrm{N})$ stage. This can affect the expected prognosis of the disease and thus have consequences for the decision regarding adjuvant therapy.

Based on these considerations, it becomes clear that the lymph node yield plays a major role in decisions regarding the therapy of malignant tumor diseases. The existing evidence on the effect of neoadjuvant therapy on the lymph node yield will be summarized in this systematic review with meta-analysis. Its primary aim is to compare the lymph node yield during oncological resection of a carcinoma of the esophagus, stomach, pancreas, and rectum after neoadjuvant chemotherapy, radiotherapy or chemoradiotherapy with the lymph node yield during upfront resection. The secondary aim is to compare the lymph node yield in defined subgroups of patients (neoadjuvant chemotherapy, neoadjuvant radiotherapy, neoadjuvant chemoradiotherapy, and patients with carcinoma of the esophagus, stomach, pancreas, and rectum) and to assess secondary outcomes such as the number of metastatic lymph nodes, the lymph node ratio, the incidence of postoperative complications, and postoperative survival time.

This report contains the protocol of the review.

\section{Methods/design}

This protocol is reported according to the recommendations of PRISMA-P 2015 statement (13). The pertinent checklist can be found in additional file 1 .

\section{Eligibility criteria}


The eligibility criteria for studies to be included in the systematic review and meta-analysis are as follows:

- They include patients in whom a carcinoma of the esophagus, stomach, pancreas, or rectum was resected oncologically, i.e., with systematic lymphadenectomy.

- They include at least one group of patients who underwent neoadjuvant therapy (chemotherapy, radiotherapy, or chemoradiotherapy) prior to surgery and one group of patients who underwent upfront surgery (surgery without prior neoadjuvant therapy).

- They report the lymph node yield (number of resected lymph nodes) for study participants.

- There is no limitation regarding study design if the criteria named above are met.

- Abstract and full text of the study are available in English, German, Russian, Italian, Spanish or French.

\section{Information sources}

The electronic literature databases PubMed and Cochrane Library will be searched through their respective online search engines using a defined search strategy (see below). The search will be performed on studies published between database inception and the cutoff date October 8, 2020. Moreover, the reference lists of included articles will be manually searched.

\section{Search strategy}

The search strategy used for the databases can be found in additional file 2 .

\section{Data management}

The abstracts of the publications identified with the search strategy will be uploaded into the web application Rayyan QCRI (14), in which study selection will be performed as described below. Data extracted from the single studies will be stored in a standardized spreadsheet and will subsequently be transferred into the review software RevMan 5.3. (15)

\section{Selection process}

Two independent reviewers will read the abstracts of the studies identified by the literature search. The reviewers use the abstract to determine whether the studies meet the inclusion criteria. If a final assessment is not possible based on the abstract alone, the assessment will be based on the full text of the publication. A study is included or excluded from the systematic review if both reviewers unanimously decide. If no agreement can be reached between the two independent reviewers, a third, independent reviewer will be used as arbiter in the selection process. Duplicates and multiple reports of the same study will be identified and excluded or collated, so that each study rather than each report will be the unit of interest in the review. The record selection process will be recorded in sufficient detail to complete a PRISMA flow diagram.

\section{Data collection process}


A standardized data collection form will be used for the collection of study characteristics and outcome data. The form will be piloted on at least one study included in the review. One review author will independently extract study characteristics and results from included studies. The following study characteristics and results will be extracted.

\section{Data items}

From the full texts of the selected publications, the following data will be collected for the overall study population and the defined subgroups, if available from the publication:

- General information on the publication: title, authors, date of publication, status of publication, journal in which the manuscript was published, language of the publication, funding of the study.

- Study design

- Disease for which the study participants were treated (carcinoma of the esophagus, stomach, pancreas, or rectum)

- Patient characteristics: sex, age, category in the ASA physical status classification system (16), ECOG Performance Status (17)

- Pretherapeutic cTNM stage

- Description of the surgical approach(es)

- Possible neoadjuvant therapy:

Chemotherapy

Radiotherapy

Chemoradiotherapy

Regimen and number of cycles as well as dosage of chemotherapeutic drugs

- Lymph node yield during resection (total number of histopathologically identified lymph nodes in the resection specimen)

- Positive lymph nodes (number of lymph nodes in the resection specimen with histopathological confirmation of tumor invasion)

- Lymph node ratio (number of positive lymph nodes divided by the lymph node yield)

- Duration of the operation

- Postoperative complications (if available, according to the Clavien-Dindo classification (18))

- pTNM stage (from resection specimen)

- Overall survival time

- Disease-free survival time

\section{Outcomes and prioritization}

A meta-analysis will be conducted for the following outcomes: 
Primary outcome

- Lymph node yield during resection

Secondary outcomes

- Positive lymph nodes

- Lymph node ratio

- Duration of the operation

- Postoperative complications (if available, according to the Clavien-Dindo classification (18))

- pTNM stage

- Overall survival time

- Disease-free survival time

\section{Risk of bias in individual studies}

The risk of bias of the individual studies will be estimated according to the respective study design.

For non-randomized studies, the ROBINS-I tool developed by the Cochrane Collaboration will be used. A full description of this tool can be found in the Cochrane Handbook (19). Prior to assessment, an emulated ideal randomized controlled trial aiming to answer the research question will be conceived. This trial will serve as a reference against which the actual studies are compared regarding their risk of bias in the single domains. The following domains of bias will be considered:

\section{Pre-intervention domains}

- Bias due to confounding

- Bias in selection of participants into the study

\section{At-intervention domain}

- Bias in classification of interventions

\section{Post-intervention domains}

- Bias due to deviations from intended interventions

- Bias due to missing data

- Bias in measurement of the outcome

- Bias in selection of the reported result

Specifically for the present meta-analysis, the following confounding domains will be addressed: pretherapeutic tumor stage, pretherapeutic physical status, age. These are supposed to predict whether a 
study participant undergoes neoadjuvant therapy or not. A specific co-intervention, which could be related to the intervention received and which is at the same time prognostic for the outcome of interest, is surgical approach. This will also be considered specifically as potential source of confounding bias.

Table 1

Bias Judgement (19)

\begin{tabular}{|lll|}
\hline $\begin{array}{l}\text { Overall } \\
\text { risk-of- } \\
\text { bias } \\
\text { judgement }\end{array}$ & Interpretation & Criterion \\
\hline $\begin{array}{l}\text { Low risk } \\
\text { of bias }\end{array}$ & $\begin{array}{l}\text { The study is comparable to a well-performed } \\
\text { randomized trial. }\end{array}$ & $\begin{array}{l}\text { The study is judged to be at low risk } \\
\text { of bias for all domains for this } \\
\text { result. }\end{array}$ \\
$\begin{array}{l}\text { Moderate } \\
\text { risk of } \\
\text { bias }\end{array}$ & $\begin{array}{l}\text { The study appears to provide sound evidence } \\
\text { for a non-randomized study but cannot be } \\
\text { considered comparable to a well-performed } \\
\text { randomized trial. }\end{array}$ & $\begin{array}{l}\text { The study is judged to be at low or } \\
\text { moderate risk of bias for all } \\
\text { domains. }\end{array}$ \\
$\begin{array}{l}\text { Serious } \\
\text { risk of } \\
\text { bias }\end{array}$ & The study has one or more important problems. & $\begin{array}{l}\text { The study is judged to be at serious } \\
\text { risk of bias in at least one domain, } \\
\text { but not at critical risk of bias in any } \\
\text { domain. }\end{array}$ \\
\hline
\end{tabular}

For each domain, the tool foresees 'signaling questions' whose response options are "yes" / "probably yes" / "probably no" / "no" / "no information". Based on the responses, the risk of bias for each domain will be judged as 'low', 'moderate', 'serious', 'critical' or 'no information'. From the risk of bias for the single domains, an overall risk of bias for the study will be ascertained according to Table 1.

For randomized trials, the RoB 2 tool developed by the Cochrane Collaboration will be used. A full description of this tool can be found in the Cochrane Handbook (20). The following domains of bias will be considered:

- Bias arising from the randomization process

- Bias due to deviations from intended interventions

- Bias due to missing outcome data

- Bias in measurement of the outcome

- Bias in selection of the reported result

For each domain, the tool foresees 'signaling questions' whose response options are "yes" / "probably yes" / "probably no" / "no" / "no information". Based on the responses, the risk of bias for each domain will be judged as 'low', 'moderate', 'serious', 'critical' or 'no information'. From the risk of bias for the single domains, an overall risk of bias for the study will be ascertained according to Table 2 : 
Table 2

Bias Judgement (20)

\section{Overall risk-of- Criteria bias judgement}

Low risk of bias The trial is judged to be at low risk of bias for all domains for this result.

Some concerns The trial is judged to raise some concerns in at least one domain for this result, but not to be at high risk of bias for any domain.

High risk of bias

The trial is judged to be at high risk of bias in at least one domain for this result.

Or

The trial is judged to have some concerns for multiple domains in a way that substantially lowers confidence in the result.

The overall risk of bias determined for the single studies (low, moderate / some concerns, high / serious) will be used to include the respective study in subsequent sensitivity analyses (see below).

\section{Data synthesis}

The primary outcome (lymph node yield) will be reported separately for the intervention group (neoadjuvant therapy) and control group (upfront surgery) as weighted mean with standard deviation. If studies do not report mean and standard deviation, these will be calculated using the methods described by the Cochrane Collaboration and Hozo et al. $(21,22)$. The groups will be compared using the weighted mean difference (and relative difference of standard deviation) for which $95 \%$ confidence intervals will be calculated. A forest plot will be drawn. The same analysis of the primary outcome will be done for the defined subgroups of patients with neoadjuvant chemotherapy versus neoadjuvant radiotherapy versus neoadjuvant chemoradiotherapy and esophageal versus gastric versus pancreatic versus rectal cancer.

The secondary outcomes number of positive lymph nodes, lymph node ratio and duration of the operation will be assessed in the same way. The secondary outcome postoperative complications will be dichotomized (grade 1 and 2 versus grade $3 a$ and higher according to the Clavien-Dindo classification). The incidence of severe complications (grade $3 a$ and higher) per group will be determined and compared using the chi ${ }^{2-}$ test and a forest plot. Rates for the secondary outcomes overall and disease-free survival at 1,3 , and 5 years will be compared using weighted rates and a forest plot. The histopathological tumor stage (pTNM) will be qualitatively described for the groups.

Sensitivity analyses will be conducted according to ascertained risk of bias as described above. For these, all studies with a high/serious risk of bias will be excluded and the analyses of the primary outcome, as described above, will be conducted.

The $\mathrm{I}^{2}$ statistic, $\mathrm{p}$ value from $\mathrm{chi}^{2}$ test, and the between-study heterogeneity $\mathrm{T}^{2}$ will be used to assess heterogeneity among the trials in each analysis. If substantial heterogeneity (greater than $50 \%$ ) is identified, reasons for this will be sought by performing subgroup analyses considering the specified 
subgroups and the causes of heterogeneity. Heterogeneity will also be assessed by evaluating whether there is good overlap of confidence intervals. Any statistical heterogeneity will be taken into account when interpreting the results.

To assess possible publication bias, if the number of included studies is sufficient, we will create a funnel plot using the primary outcome and evaluate funnel asymmetry with Begg's and Egger's tests $(23,24)$ with respect to continuous data or Peters' test with respect to binary data (25).

\section{Assessing the strength of the body of evidence}

A 'Summary of findings' table will be created using the five GRADE considerations (study limitations, consistency of effect, imprecision, indirectness, and publication bias) to assess the quality of the body of evidence based on the studies that contributed data to the meta-analyses for each outcome, classifying it as high, moderate, low or very low. The methods and recommendations described in Chap. 14 of the Cochrane Handbook for Systematic Reviews of Interventions will be used. (26)

\section{Discussion}

This systematic review with meta-analysis has the aim of summarizing all available evidence regarding the oncological surrogate marker lymph node yield in patients with esophageal, gastric, pancreatic, and rectal carcinoma undergoing surgery after neoadjuvant treatment compared to those operated without neoadjuvant therapy. Moreover, several clinically relevant secondary outcomes will be assessed and compared between the two groups. The findings of the analysis can yield information if neoadjuvant therapy is associated with a reduced or increased lymph node yield, which is an oncological surrogate marker. Moreover, it can provide hints for possible clinical implications of such a reduced or increased yield.

\section{Abbreviations}

ASA American Society of Anesthesiologists

ECOG Eastern Cooperative Oncology Group

ESMO European Society for Medical Oncology

GRADE Grading of Recommendations, Assessment, Development and Evaluations

PRISMA-P Preferred reporting items for systematic review and meta-analysis protocols

ROBINS-I Risk Of Bias In Non-randomized Studies of Interventions

TNM Tumor Node Metastasis stage

\section{Declarations}


Ethics approval and consent to participate: The ethical committee of the Medical Faculty of the MartinLuther University Halle-Wittenberg waived this study from the need for ethical approval because no data from individual patients will be used.

Consent for publication: Not applicable.

Availability of data and materials: Not applicable.

Competing interests: The authors declare that they have no competing interests.

Funding: This study is supported by intramural research funds of the Medical Faculty of the Martin Luther University Halle-Wittenberg (Advanced Clinician Scientist Program).

Authors' contributions: JK conceived of the study rationale and the research questions. UR, NM and JF developed the study methods and drafted the study protocol. All authors have critically revised the study protocol and approved its final submitted version.

Acknowledgments: Not applicable.

\section{References}

1. Brierley JD, Gospodarowicz MK, Wittekind C, editors. The TNM classification of malignant tumours. 8. Oxford: Wiley Blackwell; 2017.

2. Leitlinie Kolorektales Karzinom. http://www.leitlinienprogramm-onkologie.de/leitlinien/kolorektaleskarzinom. Accessed 10 August 2020.

3. Glynne-Jones R, Wyrwicz L, Tiret E, Brown G, Rödel C, Cervantes A, et al. Rectal cancer: ESMO Clinical Practice Guidelines for diagnosis, treatment and follow-up. Ann Oncol. 2017;28(suppl_4):iv22-40.

4. Vogel JD, Eskicioglu C, Weiser MR, Feingold DL, Steele SR. The American Society of Colon and Rectal Surgeons Clinical Practice Guidelines for the Treatment of Colon Cancer. Dis Colon Rectum. 2017;60:999-1017.

5. Doll D, Gertler R, Maak M, et al. Reduced lymph node yield in rectal carcinoma specimen after neoadjuvant radiochemotherapy has no prognostic relevance. World J Surg. 2009;33:340-7.

6. Mechera R, Schuster T, Rosenberg R, Speich B. Lymph node yield after rectal resection in patients treated with neoadjuvant radiation for rectal cancer: A systematic review and meta-analysis. Eur $\mathrm{J}$ Cancer. 2017;72:84-94.

7. Miller ED, Robb BW, Cummings OW, Johnstone PA. The effects of preoperative chemoradiotherapy on lymph node sampling in rectal cancer. Dis Colon Rectum. 2012;55:1002-7.

8. Baxter NN, Morris AM, Rothenberger DA, Tepper JE. Impact of preoperative radiation for rectal cancer on subsequent lymph node evaluation: a population-based analysis. Int J Radiat Oncol Biol Phys. 2005;61:426-31. 
9. Ha YH, Jeong SY, Lim SB, et al. Influence of preoperative chemoradiotherapy on the number of lymph nodes retrieved in rectal cancer. Ann Surg. 2010;252(2):336-40.

10. Fajardo LF. Effects of ionizing radiation on lymph nodes. A review. Front Radiat Ther Oncol. 1994;28:37-45.

11. Shvero J, Koren R, Marshak G, et al. Histological changes in the cervical lymph nodes after radiotherapy. Oncol Rep. 2001;8:909-11.

12. Miller ED, Robb BW, Cummings OW, Johnstone PA. The effects of preoperative chemoradiotherapy on lymph node sampling in rectal cancer. Dis Colon Rectum. 2012;55:1002-7.

13. Moher $D$, Shamseer $L$, Clarke $M$, et al. Preferred reporting items for systematic review and metaanalysis protocols (PRISMA-P) 2015 statement. Syst Rev. 2015;4:1.

14. Ouzzani M, Hammady H, Fedorowicz Z, et al. Rayyan - a web and mobile app for systematic reviews. Syst Rev. 2016;5:210.

15. Review Manager (RevMan). [Computer program]. Version 5.3. Copenhagen: The Nordic Cochrane Centre, The Cochrane Collaboration; 2014.

16. Abouleish N, Leib ML, Cohen NH. ASA Provides Examples to Each ASA Physical Status Class". ASA Newsletter. 2015;79:38-49.

17. Oken MM, Creech RH, Tormey DC, Horton J, Davis TE, McFadden ET, Carbone PP. Toxicity and response criteria of the Eastern Cooperative Oncology Group. Am J Clin Oncol. 1982;5:649-55.

18. Dindo D, Demartines N, Clavien PA. Classification of surgical complications: a new proposal with evaluation in a cohort of 6336 patients and results of a survey. Ann Surg. 2004;240:205-13.

19. Sterne JA, Hernán MA, McAleenan A, Reeves BC, Higgins JPT. Assessing risk of bias in a nonrandomized study. In: Higgins JPT, Thomas J, Chandler J, Cumpston M, Li T, Page MJ, Welch VA, editors. Cochrane Handbook for Systematic Reviews of Interventions version 6.1 [Internet]. Cochrane, 2020 [cited 2020 Feb 16]. Chapter 8. Available from:

https://training.cochrane.org/handbook/current/chapter-25.

20. Higgins JP, Savović J, Page MJ, Elbers RG, Sterne JA. Assessing risk of bias in a randomized trial. In: Higgins JPT, Thomas J, Chandler J, Cumpston M, Li T, Page MJ, Welch VA, editors. Cochrane Handbook for Systematic Reviews of Interventions version 6.1 [Internet]. Cochrane, 2020 [cited 2020 Feb 16]. Chapter 8. Available from: https://training.cochrane.org/handbook/current/chapter-08.

21. Green S, Higgins J, Alderson P, Clarke M, Mulrow C, Oxman A. Cochrane handbook for systematic reviews of interventions. West Sussex: John Wiley \& Sons Ltd.; 2008.

22. Hozo SP, Djulbegovic B, Hozo I. Estimating the mean and variance from the median, range, and the size of a sample. BMC Med Res Methodol. 2005;5:13.

23. Begg CB, Mazumdar M. Operating characteristics of a rank correlation test for publication bias. Biometrics. 1994;50:1088-101.

24. Egger M, Davey Smith G, Schneider M, Minder C. Bias in meta-analysis detected by a simple, graphical test. BMJ. 1997;315(7109):629-34. 
25. Peters JL, Sutton AJ, Jones DR, Abrams KR, Rushton L. Comparison of two methods to detect publication bias in meta-analysis. JAMA. 2006;295:676-80.

26. Schünemann HJ, Higgins JPT, Vist GE, Glasziou P, Akl EA, Skoetz N, Guyatt GH. Completing 'Summary of findings' tables and grading the certainty of the evidence. In: Higgins JPT, Thomas J, Chandler J, Cumpston M, Li T, Page MJ, Welch VA, editors. Cochrane Handbook for Systematic Reviews of Interventions version 6.1 [Internet].

27. Cochrane. 2020 [cited 2020 Feb 16]. Chapter 14. Available from: https://training.cochrane.org/handbook/current/chapter-14.

\section{Supplementary Files}

This is a list of supplementary files associated with this preprint. Click to download.

- Additionalfile1PRISMAPchecklist003.docx

- Additionalfile2searchstrategy.docx 